\title{
Is there an interest to use deuteron beams to produce non-conventional radionuclides?
}

\author{
Cyrille Alliot ${ }^{1,2}$, Nadia Audouin ${ }^{1}$, Jacques Barbet ${ }^{1,2}$, Anne-Cecile Bonraisin ${ }^{1}$, \\ Valérie Bossé ${ }^{1,2}$, Cécile Bourdeau ${ }^{1}$, Mickael Bourgeois ${ }^{1,2}$, Charlotte Duchemin ${ }^{3}$, \\ Arnaud Guertin $^{3}$, Ferid Haddad ${ }^{1,3 *}$, Sandrine Huclier-Markai ${ }^{3}$, Rabah Kerdjoudj ${ }^{3}$, \\ Johan Laizé ${ }^{1}$, Vincent Métivier ${ }^{3}$, Nathalie Michel ${ }^{1,3}$, Marcel Mokili, ${ }^{1,3}$, Mickael Pageau ${ }^{1}$ and \\ Aurélien Vidal ${ }^{1}$
} ${ }^{1}$ GIP Arronax, Saint-Herblain, France, ${ }^{2}$ CRCNA, CNRS, INSERM, Université de Nantes, Nantes, France, ${ }^{3}$ Subatech, EMN-
IN2P3/CNRS, Université de Nantes, Nantes, France

OPEN ACCESS

Edited by: Anil Kumar Mishra,

Defence Research Development Organization, India

Reviewed by:

Puja Panwar Hazari,

Institute of Nuclear Medicine and Allied Sciences (INMAS), India

Maria Mathew D'Souza,

Institute of Nuclear Medicine and

Allied Sciences (INMAS), India

*Correspondence:

Ferid Haddad,

GIP ARRONAX, 1 rue Aronnax, CS

10112, Saint-Herblain Cedex 44817 ,

France

haddad@cyclotron-nantes.fr

Specialty section:

This article was submitted to Nuclear

Medicine, a section of the journal

Frontiers in Medicine

Received: 10 April 2015

Accepted: 27 April 2015

Published: 11 May 2015

Citation:

Alliot C, Audouin N, Barbet J, Bonraisin A-C, Bossé V, Bourdeau C, Bourgeois M, Duchemin C, Guertin A,

Haddad F, Huclier-Markai S, Kerdjoudj R, Laizé J, Métivier V, Michel N, Mokili M, Pageau M and

Vidal A (2015) Is there an interest to use deuteron beams to produce non-conventional radionuclides? Front. Med. 2:31.

doi: 10.3389/fmed.2015.00031
With the recent interest on the theranostic approach, there has been a renewed interest for alternative radionuclides in nuclear medicine. They can be produced using common production routes, i.e., using protons accelerated by biomedical cyclotrons or neutrons produced in research reactors. However, in some cases, it can be more valuable to use deuterons as projectiles. In the case of Cu-64, smaller quantities of the expensive target material, Ni-64, are used with deuterons as compared with protons for the same produced activity. For the Sc-44m/Sc-44g generator, deuterons afford a higher Sc-44m production yield than with protons. Finally, in the case of Re-186g, deuterons lead to a production yield five times higher than protons. These three examples show that it is of interest to consider not only protons or neutrons but also deuterons to produce alternative radionuclides.

Keywords: radionuclide production, deuteron, theranostic, copper-64, rhenium-186, scandium-44

\section{Introduction}

Radionuclides are used in nuclear medicine for both imaging and/or therapy (1). For the last two decades, positron emission tomography (PET) and molecular radiotherapy have developed rapidly. Fluorine-18 and especially F-18 fluorodeoxyglucose, FDG, is the main driving force for PET imaging (1) whereas yttrium-90 and lutetium-177 have shown promising clinical results for antibody or peptide labeling.

Recently, the concept of theranostics, which is a treatment strategy that combines therapy and diagnosis, has emerged. By doing imaging prior to the treatment, it is possible to select patients that will respond to a given treatment, to make dosimetry prior to therapy, and to define the activity to inject. Treatment efficacy may also be assessed. To achieve this aim, several types of isotopes can be selected: radionuclides that possess radiations for both imaging and therapy, as for example Sn$117 \mathrm{~m}$, pairs of radionuclide of the same element, like I-124 (for imaging) and I-131(for treatment), or radionuclides with comparable chemical properties like Tc-99m (for imaging) and Re-186g (for treatment).

The theranostic approach has renewed the interest for unconventional radionuclides in nuclear medicine and many radioisotopes with different properties (half-life, beta energy, gamma emissions) are under study for either imaging or therapeutic use. These isotopes are mostly produced using biomedical cyclotrons, which usually accelerate low-energy protons, or nuclear reactors, depending on the radiation of interest. In all cases, it is of great importance to get high-specific activity. 
The aim of this paper is to illustrate the interest of using deuterons as projectiles to produce alternative radionuclides for medical applications. For that purpose, three examples are presented. These examples have been chosen according to the fact that actual productions have been made. After a description of the Arronax facility, which produces two out of the three isotopes on a regular basis, and intends, in the future, to explore the possibility to produce the last one, each isotope is presented. The added value of using deuterons as projectiles is presented for each of them.

\section{The Arronax Facility}

\section{The Cyclotron}

The Accelerator for Research in Radiochemistry and Oncology in Nantes Atlantique (Arronax) (2) cyclotron has turned into operation in February 2011. It accelerates both positive $\left(\mathrm{HH}^{+}\right.$, $\mathrm{He}^{++}$) and negative ions $\left(\mathrm{H}^{-}, \mathrm{D}^{-}\right)$up to $70 \mathrm{MeV}$ and delivers up to $750 \mu \mathrm{Ae}$ (two beams of $375 \mu \mathrm{Ae}$ ) of protons. This has been tested successfully for $24 \mathrm{~h}$ in a row during the commissioning phase. Arronax can deliver up to $70 \mu \mathrm{Ae}$ of alpha-particles. The capabilities of the cyclotron are summarized in Table 1.

Negative ions are extracted using the stripper foil technique. This technique allows beam extraction with $99 \%$ efficiency within a large range of incident energy by changing the radial position of the foil. Here, the proton beam can be extracted from 30 to $70 \mathrm{MeV}$. Arronax is equipped with two such devices diametrically opposed. This gives the ability to deliver two beams with different energies and intensities (up to $350 \mu \mathrm{Ae}$ each for protons) at the same time. Same feature can be made for the deuteron beam but with a lower intensity (see Table $\mathbf{1}$ ).

Positive ions are extracted using an electromagnetic septum. In this case, only one beam output is available at a fixed energy of $68 \mathrm{MeV}$ for alpha-particles (extraction efficiency of $83 \%$ ) and $17 \mathrm{MeV}$ for $\mathrm{HH}^{+}$ions (extraction efficiency of $80 \%$ ). This last possibility provides a proton beam at lower energy than that of $\mathrm{H}^{-}$particles without the use of a beam energy degrader.

\section{The Surrounding Facility}

Arronax can deliver a particle beam in six experimental vaults named AX, A1, A2, P1, P2, and P3 (see Figure 1). Due to the extraction method, which is different for negative and positive ions, protons and deuterons are available in all experimental vaults, whereas alpha-particles and $\mathrm{HH}^{+}$generating protons are available only in vaults $\mathrm{A} 1, \mathrm{~A} 2$, and $\mathrm{AX}$.

Vaults A1, A2, P2, and P3, which are dedicated to radionuclide production, are equipped with irradiation stations and remote pneumatic transfer systems (rabbit system) of the irradiated materials from the beam lines into the hot cells. This system insures the

TABLE 1 | Characteristics of the beams available at Arronax.

\begin{tabular}{lcccc}
\hline Beam & $\begin{array}{c}\text { Accelerated } \\
\text { particles }\end{array}$ & $\begin{array}{c}\text { Energy } \\
\text { range }(\mathbf{M e V})\end{array}$ & $\begin{array}{c}\text { Intensity } \\
(\mu \mathrm{Ae})\end{array}$ & $\begin{array}{c}\text { Number of simultaneous } \\
\text { extracted beams }\end{array}$ \\
\hline Protons & $\mathrm{H}^{-}$ & $30-70$ & $<375$ & 2 \\
& $\mathrm{HH}^{+}$ & Fixed 17 & $<50$ & 1 \\
Deuterons & $\mathrm{D}^{-}$ & $15-35$ & $<50$ & 2 \\
$\alpha$-Particles & $\mathrm{He}^{++}$ & Fixed 68 & $<70$ & 1
\end{tabular}

vacuum tightness in the beam lines and allows the connection to a water cooling system during irradiation. It can handle different types of targets depending on the desired radionuclide. We have developed two different designs. The first one accommodates a target tilted at $15^{\circ}$ with respect to beam axis with one face of the target placed in vacuum and the other one cooled with water. The other type of rabbit can accommodate encapsulated targets placed at $90^{\circ}$ with respect to the beam axis, which are water cooled all over their surface. A 4-fingers collimator is placed in front of the target station to center the beam and to limit its diameter.

In vault $\mathrm{P} 1$, a high power accelerator-driven neutron source is installed (3).

The largest vault, AX, is devoted to radiochemistry, physics, and radiobiology experiments as well as advanced student training. The beam line entering this vault can be sent in three different subbeam lines at the end of which experimental devices are placed. One of the beam lines is directed to a pit providing a vertical beam, which is of great interest for the irradiation of chemical solutions and cells for radiolysis or radiobiology experiments. The two other lines are currently used for physics and radiolysis experiments.

In addition, several laboratories (radiochemistry, biochemistry, hot cells, radiolabeling, chemical analysis, nuclear metrology ...) are available allowing radiopharmaceuticals development and quality control of final products.

\section{Deuteron Production of Non-Conventional Radionuclides}

To produce alternative isotopes, deuteron beams can, in some cases, be more interesting than proton beams. Three examples are presented below to illustrate this fact. All the nuclear data used were extracted from the NUDAT database (4).

\section{Scandium-44}

Sc-44g has a half-life of $3.97 \mathrm{~h}$. It decays through electron capture in $5.73 \%$ of the cases and by $\beta^{+}$emission in $94.27 \%$ of the cases with a mean kinetic energy of $632 \mathrm{keV}$. It can be used for PET imaging and will be complementary to the shorter half-life ${ }^{68} \mathrm{Ga}$ $\left(T_{1 / 2}=67.71 \mathrm{~min}\right)$. It is possible to produce an excited state of Sc44 called Sc- $44 \mathrm{~m}\left(T_{1 / 2}=58 \mathrm{~h}\right)$, which decays to the ground state by gamma emission $\left(E_{\gamma}=271.24 \mathrm{keV}, I_{\gamma}=86.7 \%\right)$. It has been proven that when a molecule is labeled with Sc-44m, the Sc-44g formed by its decay remains attached to the molecule (5). This indicates that Sc-44m/Sc-44g may be used as an in vivo generator to study long biological processes such as those associated to labeled full antibodies.

Among the scandium isotopes, $\mathrm{Sc}-47$ ( $\beta^{-}$emitter) is well suited for use in targeted radionuclide therapy. There is then a potential interest to use Sc-44 for dosimetry studies before using Sc-47 for therapy, as a theranostic approach (6).

Finally, associated to Sc-44g decay, a high-energy gamma ray at $1.157 \mathrm{MeV}$ with a high probability of occurrence $(99.9 \%)$ is emitted within nanoseconds. By measuring the incident direction of this photon and the line of response of the $511 \mathrm{keV}$ photons resulting from the $\beta^{+}$decay, it is possible to get, on an event by event basis, the emitter location in three dimensions as proposed by Grignon et al. (7). With a Compton telescope using a 


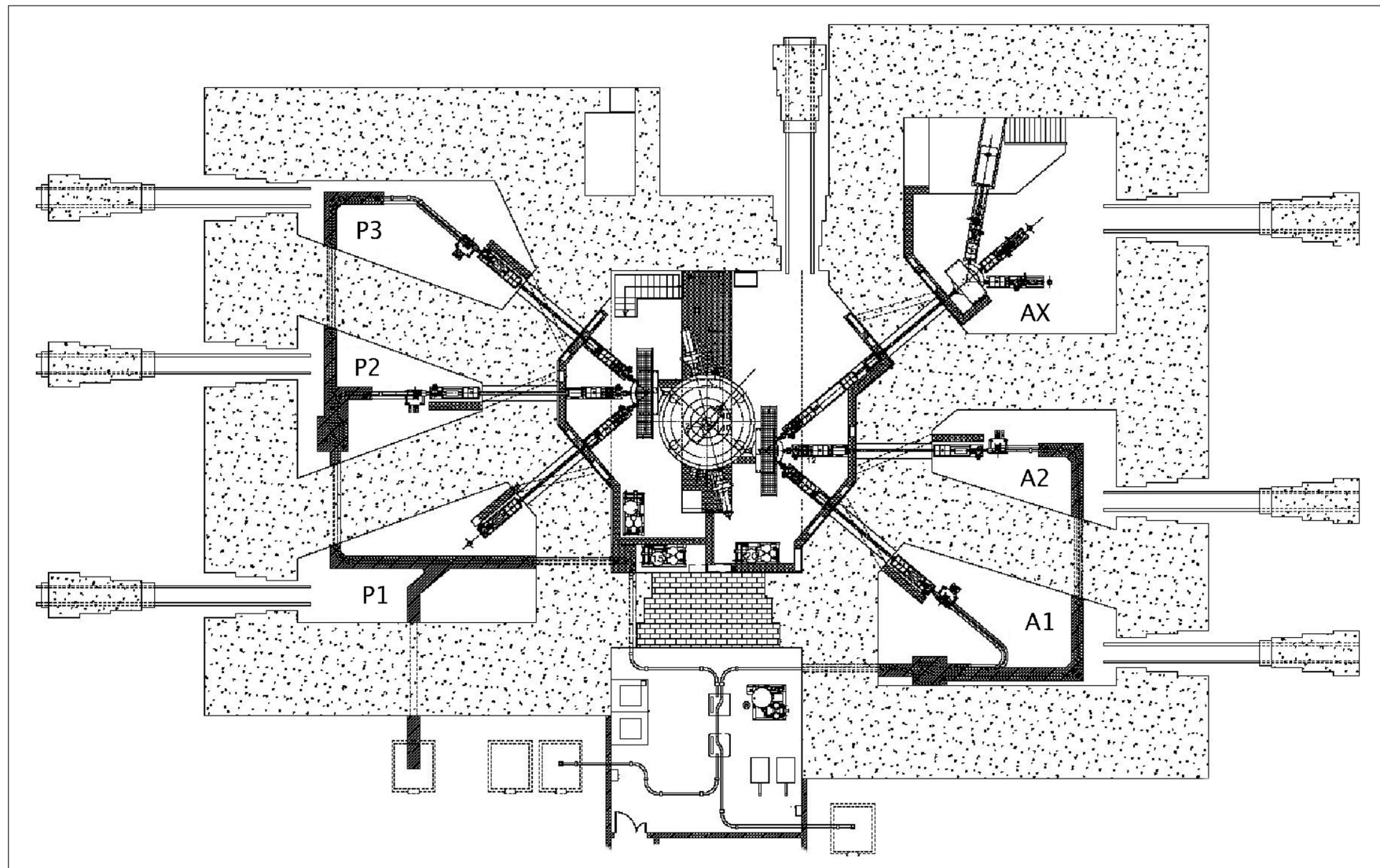

FIGURE 1 | Schematic view of the ARRONAX cyclotron and its target vaults.

new generation of cameras based on a liquid xenon time projection chamber, a spatial resolution of $2.3 \mathrm{~mm}$ has been estimated with an injected activity of $0.5 \mathrm{MBq}$ for a Sc- $44 \mathrm{~g}$ point source emitter (7).

Sc-44g and Sc- $44 \mathrm{~m}$ can be produced using enriched ${ }^{44} \mathrm{CaCO}_{3}$ as target. The use of an enriched target maximizes the scandium production and reduces the production of contaminants. Using protons, production is achieved through the ${ }^{44} \mathrm{Ca}(\mathrm{p}, \mathrm{n})$ reaction. This reaction has an energy threshold of $4.536 \mathrm{MeV}$. One set of cross-section data point exists (8) and can be used to estimate the production yield. On Figure 2, the evolution of the production cross-section of Sc-44 as a function of the proton incident energy is presented. Data of Levkovskij (8) have been normalized by a factor 0.8 due to a monitor problem (9). The maximum value of the cross-section is obtained for $12 \mathrm{MeV}$ protons. The production of the Sc-43, via the reaction $(p, 2 n)$, starts at $14,458 \mathrm{MeV}$. By selecting proton incident energy below this value, it is possible to prevent the production of Sc-43 although this isotope is an attractive candidate for conventional PET imaging.

As stated before, Sc- $44 \mathrm{~m}$ is also produced during the irradiation. The production cross-section of the metastable state changes with the incident energy of the proton energy. The ratio of the production cross-section of the metastable state over the ground state has been measured by Sachdev et al. (10) for the (p,n) reaction and its values are presented in Table 2 . The measurements made by Levkovskij (8) give values of the same order of magnitude. In the energy range of interest, the Sc- 44 m will represent $<15 \%$ of

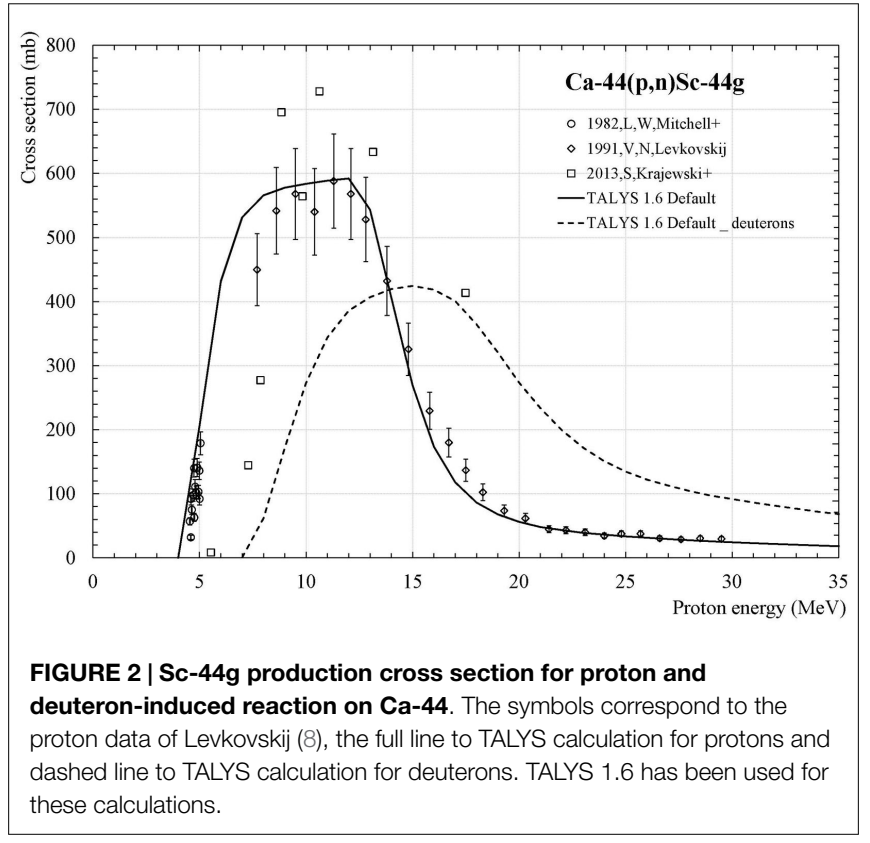

the produced $\mathrm{Sc}-44 \mathrm{~g}+\mathrm{Sc} 44 \mathrm{~m}$ atoms. It has to be kept in mind that, experimentally, we have access to the activity and not to the number of produced isotope. Due to the large difference in half lives, activity ratio will be much smaller (divide by a factor of $\sim 15$ ). 
TABLE 2 | Evolution of the cross-section ratio as a function of the proton incident energy (10)

\begin{tabular}{|c|c|c|c|c|c|c|c|c|c|c|c|c|c|}
\hline $\mathrm{E}(\mathrm{MeV})$ & 6 & 9 & 12 & 15 & 18 & 21 & 24 & 30 & 40 & 50 & 60 & 72 & 85 \\
\hline$\sigma(\mathrm{Sc}-44 \mathrm{~m}) /(\sigma(\mathrm{Sc}-44 \mathrm{~g})$ & 0.015 & 0.063 & 0.090 & 0.140 & 0.20 & 0.24 & 0.23 & 0.19 & 0.16 & 0.14 & 0.15 & 0.16 & 0.17 \\
\hline
\end{tabular}

Another parameter that affects this ratio is the irradiation time. The saturation is reached earlier for Sc-44g than Sc- $44 \mathrm{~m}$.

An alternative production route is to use deuterons as projectiles through the ${ }^{44} \mathrm{Ca}(\mathrm{d}, 2 \mathrm{n})$ reaction, which has an energy threshold equal to $6.964 \mathrm{MeV}$. We did not find cross-section data with deuterons in the literature and we have therefore chosen to use the TALYS code version 1.6 (11) to estimate the production yields in order to compare with the proton route. TALYS is a computer program for simulation of nuclear reactions induced by light projectiles on nuclei heavier than carbon. It integrates many theoretical models that predict the behavior of various observables, among which the production cross-sections, in the energy range from $1 \mathrm{keV}$ to $1000 \mathrm{MeV}$. TALYS can work with a limited number of input parameters: the type of projectile and its incident energy, the target element and its atomic mass. Default settings are assigned to each nuclear model and several parameters can be modified to better describe the data. In this work, we used the default settings.

Before using the code to obtain the $(\mathrm{d}, 2 \mathrm{n})$ cross-section values, we have compared the experimental cross-section for the reaction $(\mathrm{p}, \mathrm{n})$ measured by Levkovskij (8) with the values predicted by TALYS. The data are presented as symbols on Figure $\mathbf{2}$ whereas the TALYS values are presented as full lines on the same Figure. A good agreement is found between the data and the code values giving us confidence on the ability of this code to predict deuteron cross-section values.

TALYS was then used to estimate the Sc-44g and Sc-44m production cross-sections associated to the $(\mathrm{d}, 2 \mathrm{n})$ reaction on $\mathrm{Ca}-44$. On Figure 2, the dashed line corresponds to the Sc-44g crosssection obtained with the TALYS code. We find that the production cross-section maximum value with deuterons is comparable to the proton one. The curve has a maximum a little lower in amplitude but it is wider. Based on this curve, we see that we must be in the energy range $10-20 \mathrm{MeV}$ to optimize the production of Sc-44g with deuterons. This range is consistent with the available deuteron energy on ARRONAX. In addition, production being related to the integral of cross-section over energy, yield using deuterons will be at least equal to that obtained with protons.

TALYS can also calculate the cross-section ratio of Sc- $44 \mathrm{~m}$ over Sc-44g. The latter is presented as a function of the projectile incident energy in Figure 3. TALYS calculations for protons and deuterons are presented, respectively, as full line and dashed line. From this figure, it is clear that the values obtained with deuterons are larger than with protons. With deuterons, as much as three times more Sc- $44 \mathrm{~m}$ can be expected as compared to protons.

The last point of interest, when comparing both production routes, is related to the co-production of other isotopes. Using a proton beam and a typical enriched target, only Sc-43 will have an impact on the specific activity. The other produced isotopes are stable or have short periods (less than minutes). Sc-43 amount can be tuned by changing the proton incident energy. Below the $(\mathrm{p}, 2 \mathrm{n})$ reaction threshold, no $\mathrm{Sc}-43$ is produced.

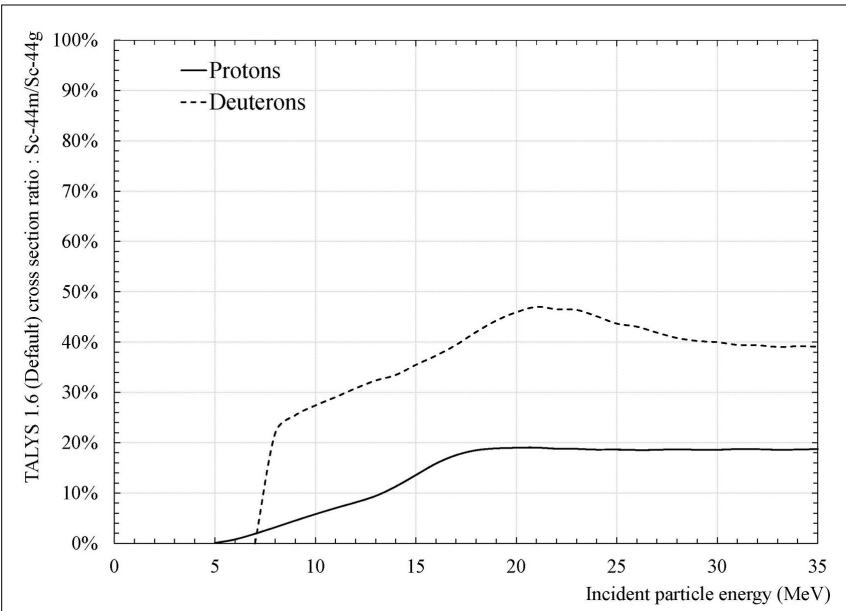

FIGURE 3 | Ratio of the cross section associated to Sc-44m over the cross-section associated to Sc-44g obtained from the TALYS code.

Using a deuteron beam and a typical enriched target, $\mathrm{Ca}-45$ $\left(T_{1 / 2}=162.61\right.$ days) will be produced via $(\mathrm{d}, \mathrm{p})$ reaction as well as $\mathrm{K}-42\left(T_{1 / 2}=12.321 \mathrm{~h}\right)$ via the $(\mathrm{d}, \alpha)$ and $\mathrm{K}-43\left(T_{1 / 2}=22.3 \mathrm{~h}\right)$ via the $(\mathrm{d}, 2 \mathrm{pn})$ reactions. These isotopes can be removed during the chemical separation process but they will put some constraints on it. The potassium isotopes produce scandium isotopes during their decay that will have an impact on the specific activity of the final product. It is then necessary to speed up the chemical process in order to keep a high-specific activity.

At Arronax, small amounts of Sc-44g and Sc- $44 \mathrm{~m}$ have been made using an irradiation station devoted to the production of low activity batches (a few millicurie at maximum). With this device, our target is placed in air, $6.6 \mathrm{~cm}$ downstream the beam line output closed by a $75 \mu \mathrm{m}$-thick kapton foil, which makes the separation between the vacuum in the line and the air in the vault. Each target is made of $500 \mathrm{mg}$ of ${ }^{44} \mathrm{CaCO}_{3}$ pressed to form an $18 \mathrm{~mm}$ diameter pellet. To prevent any contamination from the $\mathrm{CaCO}_{3}$ powder during irradiation, each target is placed inside a frame made of $75 \mu \mathrm{m}$ kapton sheets on both sides. An original chemistry has been developed using DGA chromatographic column (5) from TrisKem International SAS (France). After the chemistry, the expensive target material is recovered for reprocessing.

The target is generally irradiated with deuterons of $16.4 \mathrm{MeV}$ for $180 \mathrm{~min}$ with a beam intensity around $300 \mathrm{nA}$. Scandium is recovered as $\mathrm{ScCl}_{3}$. Typical activity for $\mathrm{Sc}-44 \mathrm{~g}$ is $90 \mathrm{MBq}$ whereas it is $1.8 \mathrm{MBq}$ for Sc-44m at the end of the chemistry step (around $4 \mathrm{~h}$ after EOB). The activity ratio is then equal to $2 \%$, which is coherent with TALYS calculations. If one except Sc- $44 \mathrm{~g}$ and Sc$44 \mathrm{~m}$, all radionuclides are below the detection limit using our HP-GE detector. Using an ICP-OES, the main stable contaminants have been identified ( $\mathrm{Al}, \mathrm{Fe}, \mathrm{Zn}$, and $\mathrm{Sc}$ ) and the total content 

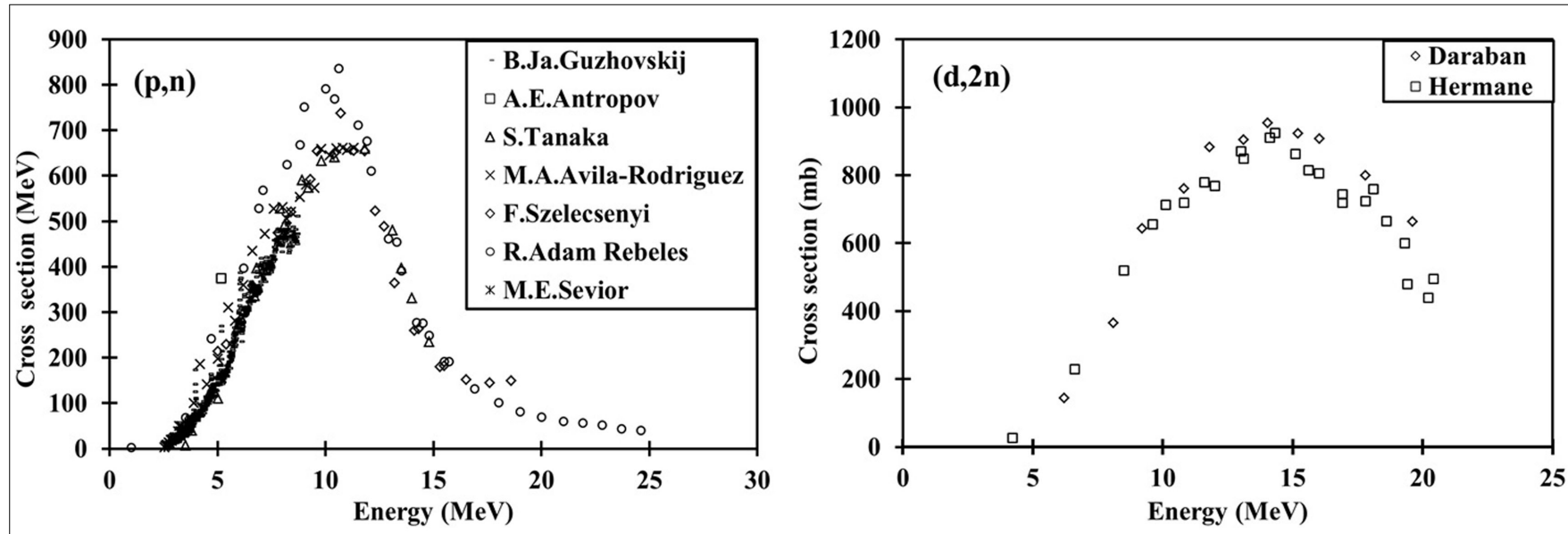

FIGURE 4 | Production cross-section as a function of the projectile energy for Ni-64(p,n)Cu-64 (left) and Ni-64(d,2n)Cu-64 (right). Inspired by Ref. (17).

is lower than $1 \mathrm{ppm}$. These numbers show that deuterons can be advantageously used to produce Sc- $44 \mathrm{~m}$ with a high-specific activity.

\section{Copper-64}

Cu-64 has a half-life of $12.7004 \mathrm{~h}$. It decays through electron capture in $44.00 \%$ of the case, by $\beta^{-}$in $38.48 \%$ with a maximum energy of $579.4 \mathrm{keV}$ and finally by $\beta^{+}$emission in $17.52 \%$ of the case with a maximum energy of $653 \mathrm{keV}$. Associated to these emissions, there is a high-energy gamma ray at $1345.75 \mathrm{keV}$ with a low probability of occurrence $(0.4749 \%)$. Considering the emitted radiations, it can be used for both therapy and PET imaging.

Several routes to produce $\mathrm{Cu}-64$ have been reported in the literature (12-16) among which the irradiation of enriched $\mathrm{Ni}$ 64 with protons is the most often used. As most (p,n) reactions, production cross-sections are high (see left plot on Figure 4). Looking at the deuteron production route, it is found that the maximum cross-section value for $(\mathrm{d}, 2 \mathrm{n})$ reaction is higher than for $(\mathrm{p}, \mathrm{n})$, reaching $800 \mathrm{mb}$ at $14 \mathrm{MeV}$. In addition, the curve evolves more slowly around the maximum for $(\mathrm{d}, 2 \mathrm{n})$ in comparison to $(\mathrm{p}, \mathrm{n})$ indicating a possible higher yield using deuterons (17). Using a $16 \mathrm{MeV}$ deuteron beam, it is possible to take full advantage of the maximum cross-section. In this condition, theoretical production yield has been calculated and compared with that obtained for a $12 \mathrm{MeV}$ proton beam (see Table 3). It shows that it is possible to produce the same amount of $\mathrm{Cu}-64$ in both cases with a target $25 \%$ thinner using deuterons.

Differences in the co-produced contaminants exist between the two production routes. On the one hand, the deuteron beam produces Ni-65 $\left(T_{1 / 2}=2.52 \mathrm{~h}\right)$ and stable $\mathrm{Cu}-65$, which are not created at all with the $(\mathrm{p}, \mathrm{n})$ reaction. On the other hand, less Co-61 $\left(T_{1 / 2}=1.65 \mathrm{~h}\right)$, which is created using proton via $(\mathrm{p}, \alpha)$, is produced with the deuteron reaction. When using a deuteron beam, the production of Ni-65 (which decays to $\mathrm{Cu}-65$ ) and $\mathrm{Cu}-65$ will have an impact on the final specific activity. In order to have some hints on their contributions, production yields have been calculated for these isotopes and for $\mathrm{Cu}-63$. When available, data
TABLE 3 | Cu-64 calculated yields for $(p, n)$ and $(d, 2 n)$ production routes.

\begin{tabular}{lcccc}
\hline Nuclear reaction & $\begin{array}{c}\text { Energy } \\
\text { range } \\
\mathbf{( M e V})\end{array}$ & $\begin{array}{c}\text { Calculated } \\
\text { yield } \\
\mathbf{( M B q} / \boldsymbol{\mu A h})\end{array}$ & $\begin{array}{c}\text { Target } \\
\text { thickness } \\
(\boldsymbol{\mu m})\end{array}$ & $\begin{array}{c}\text { Target } \\
\text { thickness } \\
\text { at } \mathbf{1 5}^{\circ}(\boldsymbol{\mu m})\end{array}$ \\
\hline $\mathrm{Ni}$-64(p,n)Cu-64 & $12-9$ & 228 & 120 & 31.05 \\
$\mathrm{Ni}-64(\mathrm{~d}, 2 \mathrm{n}) \mathrm{Cu}-64$ & $16-13$ & 206 & 90 & 23.29 \\
\hline
\end{tabular}

TABLE 4 | Direct and indirect productions of stable copper isotopes with a deuteron beam on $\mathrm{Ni}-64$

\begin{tabular}{lccc}
\hline Isotope & Half-life & $\begin{array}{c}\text { Decay } \\
\text { product }\end{array}$ & $\begin{array}{c}\text { Expected yield } \\
\text { (EOB) atoms }\end{array}$ \\
\hline${ }^{65} \mathrm{Ni}$ & $2.52 \mathrm{~h}$ & ${ }^{65} \mathrm{Cu}$ & $2.33 \mathrm{E}+12$ \\
${ }^{63} \mathrm{Cu}$ & $\mathrm{Stable}$ & - & $2.07 \mathrm{E}+11$ \\
${ }^{65} \mathrm{Cu}$ & Stable & - & $6.1 \mathrm{E}+11$ \\
& ${ }^{65} \mathrm{Ni}$ decays during irradiation & $3,3 \mathrm{E}+11$ \\
${ }^{64} \mathrm{Cu}$ & $12.7 \mathrm{~h}$ & Ni-64 & $1.31 \mathrm{E}+13$ \\
\end{tabular}

have been taken from Kinsey et al. (4). Otherwise, we have used the TALYS code with default parameters (11) to get cross-section values. In Table 4, the calculated yield values are presented for a $1 \mathrm{~h}$ irradiation at $1 \mu \mathrm{A}$.

The main stable copper contribution comes from the decay of Ni-65 either during irradiation or after. The latter contribution is strongly correlated to the time of the chemistry, the shorter the separation is, the lower the contribution is, whereas the first contribution increases with the irradiation time.

By considering the worst case in which all the Ni-65 has decayed to $\mathrm{Cu}-65$ when the chemistry is performed, we end up with 3.8 atoms of $\mathrm{Cu}-64$ for 1 atom of cold copper coming for a $1 \mathrm{~h}$ irradiation. This corresponds to a specific activity of $7.22 \mathrm{GBq} / \mathrm{nMol}$ as compared to the theoretical value for proton, which is $9.13 \mathrm{GBq} / \mathrm{nMol}$. These numbers are far above what is necessary for antibody labeling.

At our facility, the deuteron production route is used for $\mathrm{Cu}-64$ production since Arronax is able to deliver this type of particles 


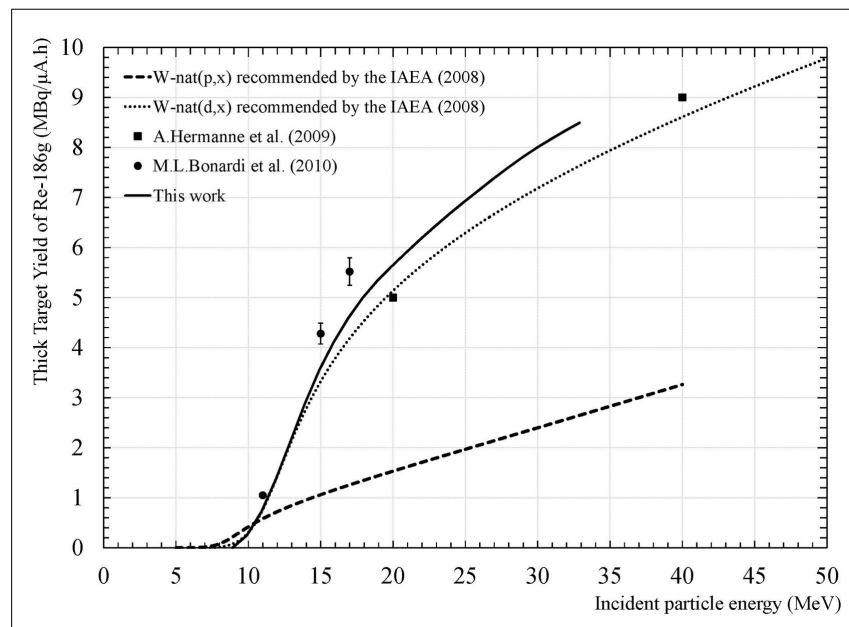

FIGURE 5 | Re-186g thick-target yield obtained from IAEA (20) data with proton and deuteron and value determined from new experimental data from Duchemin et al. (18). Symbols correspond to values extracted from Hermann et al. (21) and Bonardi et al. (22).

(see Table 3). A highly enriched (>99\%) Ni-64 target electroplated on a gold backing ( $99.99 \%$ purity) is used and an original chemistry has been developed using $\mathrm{HBr}$ on AG1X8 chromatographic column. After the chemistry, the expensive target material is recovered (recovery yield around 95\%) for reprocessing.

A typical irradiation is carried out with $50 \mu \mathrm{A}$ deuterons beam at $16 \mathrm{MeV}$ impinging a $10 \mu \mathrm{m}$-thick target for $150 \mathrm{~min}$. Copper-64 is recovered as $\mathrm{CuCl}_{2}$ with a high-radioisotopic purity (>99.90\%). Using an ICP-OES, the main contaminants have been identified as $\mathrm{Ni}, \mathrm{Cu}$, and $\mathrm{Fe}$. On a routine basis, we produce $12 \mathrm{GBq}$ at the end of bombardment. Production can be increased by using a thicker target and increasing the irradiation duration. These numbers show that deuteron can be advantageously used to produce Cu-64.

\section{Rhenium-186g}

Rhenium is a Group 7 congener of Tc and therefore, in many cases, shares remarkably similar chemical behavior to Tc. Thus, Tc-99m radiocomplexation knowledge can often be applied to rhenium radionuclides. Re-186g is a $\beta^{-}$emitter with a $137.157 \mathrm{keV} \gamma$ emission (branching ratio of $9.42 \%$ ) that can be used for imaging with SPECT. Its half-life is 3.7183 days. There is another $\beta^{-}$ emitter isotope, ${ }^{188} \mathrm{Re}$, with a half-life of 0.7 days, which has a $155.032 \mathrm{keV} \gamma$ emission suitable for imaging. In addition to their different half lives, the mean $\beta^{-}$energy of these two rhenium isotopes is different: 347 and $763 \mathrm{keV}$ for Re-186g and Re-188, respectively. Because the chemistry of rhenium is close to that of technetium, Tc-99m/Re-186g and Tc-99m/Re-188 can act as potential theranostic pairs.

Re-188 can be obtained with a high-specific activity through a W-188/Re-188 generator. Unfortunately, W-188 requires a double neutron capture to be produced limiting its production to high-flux nuclear reactor. Using nuclear reactors, it is not possible to get high specific activity Re-186g. However, it is possible to use accelerator to that purpose.
A promising production route is the $\mathrm{W}-186(\mathrm{~d}, 2 \mathrm{n})$. Its threshold energy is equal to $3.6 \mathrm{MeV}$. Deuteron with incident energy below $17.6 \mathrm{MeV}$ can be used to produce high-radionucleidic purity and high-specific activity of Re-186g. Using deuterons as projectiles, five times more Re-186g can be produced as compared to protons as projectiles (see Figure 5). Based on the calculation of Duchemin et al. (18), using an enriched W-186 target and an energy range of $3.5-17.6 \mathrm{MeV}$, a thick-target yield of $16.8 \mathrm{MBq} / \mu \mathrm{A} \cdot \mathrm{h}$ can be obtained, which corresponds to $0.5 \mathrm{Ci}$ (EOB) for $24 \mathrm{~h}$ irradiation at $50 \mu \mathrm{A}$. These numbers are in agreement with data from Xiaodong et al. (19), which have reported the production of Re$186 \mathrm{~g}$ using a $16 \mathrm{MeV}$ deuteron beam via the $(\mathrm{d}, 2 \mathrm{n})$ reaction on isotopically enriched W-186 metal powder. After chemical extraction and purification, no-carrier-added Re-186g saline solution was obtained with a radionuclidic purity $>99.9 \%$. Main isotopic impurities were Re-183 and Re-184g. The experimental thicktarget yield of Re-186g was determined to be $\sim 529 \mu \mathrm{Ci} / \mu \mathrm{A} \cdot \mathrm{h}$. These numbers show that deuteron is the projectile of choice to produce high-specific activity of Re-186g.

\section{Conclusion}

Recently, the theranostic paradigm has renewed the interest for unconventional radionuclides in nuclear medicine. Several radioisotopes with different properties (half-life, beta energy, gamma emissions) are under study for either imaging or therapeutic use. In some cases, the use of deuterons as projectiles to produce these radionuclides can be beneficial. Scandium- 44 can be used for imaging. In addition to the ground state (Sc$44 \mathrm{~g}$ ), there is a metastable state (Sc-44m) that can be used as an in vivo Sc- $44 \mathrm{~m} / \mathrm{Sc}-44 \mathrm{~g}$ generator. When using deuterons to produce these isotopes, one maximizes the production of the metastable state as compared to the ground state, which is of interest if one wants to use Sc-44 for imaging for long processes. Another example is the production of $\mathrm{Cu}-64$. It can be produced from deuteron irradiation using the same target as for proton but using a lower quantity of $\mathrm{Ni}-64$, which is a very expensive material. A theoretical study shows that the specific activity is a little lower than in the case of proton irradiation but is still high enough for medical applications. Finally, in the case of Re-186, the use of deuterons is the only viable production route since the production yield is five times higher than that obtained using protons.

At the Arronax facility, we have access to deuteron beam at the right energy and use it already for routine production of $\mathrm{Cu}-64$ and $\mathrm{Sc}-44 \mathrm{~m} / \mathrm{Sc}-44 \mathrm{~g}$. We have started to look at the Re-186g production with deuterons.

\section{Acknowledgments}

The cyclotron Arronax is supported by CNRS, INSERM, INCa, the Nantes University, the Regional Council of Pays de la Loire, local authorities, the French government, and the European Union. This work has been, in part, supported by a grant from the French National Agency for Research called "Investissements d'Avenir," Equipex Arronax-Plus ANR-11-EQPX-0004, and Labex IRON ANR-11-LABX-18-01. 


\section{References}

1. NUPECC. Nuclear Physics for Medecine, Report. Strasbourg: European Science Foundation (2014). 156 p. Available from: www.esf.org

2. Haddad F, Ferrer L, Guertin A, Carlier T, Michel N, Barbet J, et al. ARRONAX, a high-energy and high-intensity cyclotron for nuclear medicine. Eur J Nucl Med Mol Imaging (2008) 35:1377-87. doi:10.1007/s00259-008-0802-5

3. Abbas K, Buono S, Burgio N. Development of an accelerator driven neutron activator for medical radioisotope production. Nucl Instrum Methods Phys Res A (2009) 601:223-8. doi:10.1016/j.nima.2008.11.152

4. Kinsey RR, et al. The NUDAT/PCNUDAT program for nuclear data. Paper Submitted to the 9th International Symposium of Capture Gamma-Ray Spectroscopy and Related Topics. Budapest (1996).

5. Huclier-Markai S, Kerdjoudj R, Alliot C, Bonraisin AC, Michel N, Haddad $\mathrm{F}$, et al. Optimization of reaction conditions for the radiolabeling of DOTA and DOTA-peptide with $44 \mathrm{~m} / 44 \mathrm{Sc}$ and experimental evidence of the feasibility of an in vivo PET generator. Nucl Med Biol (2014) 41:e36-43. doi:10.1016/j. nucmedbio.2013.11.004

6. Srivastava SC. A bridge not too far: personalized medicine with the use of theragnostic radiopharmaceuticals. J Postgrad Med Educ Res (2013) 47(1):31-46. doi:10.5005/jp-journals- 10028-1054

7. Grignon C, Barbet J, Bardiès M, Carlier T, Chatal J-F, Couturier O. Nuclear medical imaging using $\beta+/ \gamma$ coincidences from ${ }^{44} \mathrm{Sc}$ radionuclide with liquid xenon as detection medium. Nucl Instrum Methods Phys Res A (2007) 571(1-2):142-53. doi:10.1016/j.nima.2006.10.048

8. Levkovskij B. Activation Cross Section Nuclides of Average Masses $(A=40-100)$ by Protons and Alpha-Particles with Average Energies (E=10-50 MeV), Act.Cs.By Protons and Alphas. Moscow: Levkovskij (1991).

9. Tarkanyi F, Sonck M, Hermanne A. Investigation of the ${ }^{\text {nat }} \mathrm{Mo}(\mathrm{p}, \mathrm{x})^{96 \mathrm{mg}} \mathrm{Tc}$ nuclear reaction to monitor proton beams: new measurements and consequences on the earlier reported data. Nucl Instrum Methods Phys Res B (2002) 198:183. doi:10.1016/S0168-583X(02)01528-8

10. Sachdev DR, Yaffe L. Isomer ratios for the ${ }^{44} \mathrm{Ca}(\mathrm{p}, \mathrm{n}){ }^{44} \mathrm{Sc}^{\mathrm{m}, \mathrm{g}}$ and ${ }^{85} \mathrm{Rb}(\mathrm{p}, \mathrm{n}){ }^{85} \mathrm{Sr}^{\mathrm{m}, \mathrm{g}}$ reactions. Can J Phys (1969) 47:1667-73. doi:10.1139/v69-274

11. Koning AJ, Hilaire S, Duijvestijn MC. TALYS-1.0. In: Bersillon O, Gunsing F, Bauge E, Jacqmin R, Leray S, editors. Proceedings of the International Conference on Nuclear Data for Science and Technology - ND2007, April 22-27, 2007. Nice: EDP Sciences (2008). p. 211-4.

12. Obata A, Kasamatsu S, McCarthy DW. Production of therapeutic quantities of 64Cu using a $12 \mathrm{MeV}$ cyclotron. Nucl Med Biol (2003) 30:535-9. doi:10.1016/ S0969-8051(03)00024-6

13. Hetherington EL, Sorby PJ, Camakaris J. The preparation of high specific activity copper-64 for medical diagnosis. Int J Rad Appl Instrum A (1986) 37:1242-3. doi:10.1016/0883-2889(86)90014-6
14. Kozempel J, Abbas K, Simonelli F, Zampese M, Holzwarth U, Gibson N, et al. A novel method for n.c.a. ${ }^{64} \mathrm{Cu}$ production by the ${ }^{64} \mathrm{Zn}(\mathrm{d}, 2 \mathrm{p}){ }^{64} \mathrm{Cu}$ reaction and dual ion-exchange column chromatography. Radiochim Acta (2007) 95:75-80. doi:10.1524/ract.2007.95.2.75

15. Nickles RJ. Production of a broad range of radionuclides with an $11 \mathrm{MeV}$ proton cyclotron. J Lab Comp Radiopharm (1991) 30:120.

16. Zweit J, Smith AM, Downey S. Excitation functions for deuteron induced reactions in natural nickel: production of no-carrier added $64 \mathrm{Cu}$ from enriched 64Ni target for positron emission tomography. Appl Radiat Isot (1991) 42:193. doi:10.1016/0883-2889(91)90073-A

17. Aslam MN, Sudár S, Hussain M, Malik AA, Shah HA, Qaim SM. Charged particle induced reaction cross section data for production of the emerging medically important positron emitter ${ }^{64} \mathrm{Cu}$ : a comprehensive evaluation. Radiochim Acta (2009) 97:669. doi:10.1524/ract.2009.1670

18. Duchemin C, Guertin A, Haddad F, Michel N, Métivier V. Cross section measurements of deuteron induced nuclear reactions on natural tungsten up to $34 \mathrm{MeV}$. Appl Radiat Isot (2015) 97:52-8. doi:10.1016/j. apradiso.2014.12.011

19. Xiaodong Z, Qingnuan L, Wenxin L, Rong S, Shuifa S. Production of no-carrieradded ${ }^{186} \mathrm{Re}$ via deuteron induced reactions on isotopically enriched ${ }^{186} \mathrm{~W}$. Appl Radiat Isot (2001) 54:89-92. doi:10.1016/S0969-8043(00)00268-2

20. Qaim SM, Tárkányi F, Capote R. Nuclear Data for the Production of Therapeutic Radionuclides. IAEA Technical Report Series 473. Vienna: IAEA (2011). 382 p.

21. Hermanne A, Daraban L, Tarkanyi F, Takacs S, Ditroi F, Ignatyuk A, et al. Excitations functions for some $\mathrm{W}$, Ta and $\mathrm{Hf}$ radionuclides obtained by deuteron irradiation of ${ }^{181} \mathrm{Ta}$ up to $40 \mathrm{MeV}$. Nucl Instrum Methods Phys Res B (2009) 267:3293-301. doi:10.1016/j.nimb.2009.07.008

22. Bonardi ML, Groppi F, Manenti S, Persico E, Gini L. Production study of high specific activity NCA Re-186g by proton and deuteron cyclotron irradiation. Appl Radiat Isot (2010) 68:1595-601. doi:10.1016/j.apradiso.2010.03.014

Conflict of Interest Statement: The authors declare that the research was conducted in the absence of any commercial or financial relationships that could be construed as a potential conflict of interest.

Copyright (C) 2015 Alliot, Audouin, Barbet, Bonraisin, Bossé, Bourdeau, Bourgeois, Duchemin, Guertin, Haddad, Huclier-Markai, Kerdjoudj, Laizé, Métivier, Michel, Mokili, Pageau and Vidal. This is an open-access article distributed under the terms of the Creative Commons Attribution License (CC BY). The use, distribution or reproduction in other forums is permitted, provided the original author (s) or licensor are credited and that the original publication in this journal is cited, in accordance with accepted academic practice. No use, distribution or reproduction is permitted which does not comply with these terms. 